\title{
Interplay of Binding Stoichiometry and Recognition Specificity for the Interaction of MBD2b Protein and Methylated DNA Revealed by Affinity Capillary Electrophoresis Coupled with Laser-Induced Fluorescence Analysis
}

\author{
Dandan Zou, Dapeng Zhang, Shengquan Liu, Bailin Zhao, and Hailin Wang* \\ State Key Laboratory of Environmental Chemistry and Ecotoxicology, Research Center for Eco-Environmental Sciences, Chinese \\ Academy of Sciences, Beijing 100085 China
}

\section{Supporting Information}

ABSTRACT: The methyl-CpG binding domain (MBD) family proteins can specifically bind methylated DNA sequences and thereby mediate gene transcription. In this study, we used neutral capillary electrophoresis coupled with laser-induced fluorescence to investigate the interactions of DNA and MBD2b, a model MBD family protein with the highest affinity. For this purpose, we synthesized 13 double-stranded oligonucleotides of varying length ( $20 \mathrm{bp}$ to $80 \mathrm{bp}$ ) and of varying methylation density. The sequences of these oligonucleotides were adapted from a frequently methylated promoter region of human $\mathrm{p} 16^{\mathrm{INK} 4 \mathrm{a}}$ gene. We demonstrate that multiple MBD2b proteins can bind to one DNA molecule with a DNA length-dependent binding stoichiometry. Each MBD2b protein can occupy 20 nucleotides in a bound DNA molecule regardless of the methylation status of DNA. By binding multiple MBD2b proteins (up to four protein molecules) to one dsDNA molecule $(80 \mathrm{bp})$, methylated and unmethylated DNA were bound at similar percentages. Although the total amount of the DNA-MBD2b complexes increases with increasing DNA length for both unmethylated and methylated DNA, the DNA-MBD2b complexes of 1:1 display more than 10-fold higher affinity for methylated DNA (e.g., 40 bp DNA) accompanying a 20-fold lower dissociation rate constant. Hence, our study clarifies for the first time that the specificity of MBD2b to methylated DNA decreases as more MBD2b monomers binding to the same region of DNA. Additionally, this study opens a new venue to improve MBD protein-based assays for detecting DNA methylation.
$\mathrm{O}$ ne of the most important epigenetic modifications in mammalian cells is DNA methylation. ${ }^{1-5}$ It occurs predominantly on $\mathrm{CpG}$ dinucleotides. Human gen promoters of about $60 \%$ are associated with $\mathrm{CpG}$ islands, which display high CpG density and are usually free of methylation in healthy cells. $^{2-4,6}$ Dynamic DNA methylation/demethylation regulates many cellular processes, including nuclear reprogramming, transcription, genomic imprinting, and X-chromosome inactivation. $^{4-9}$ Dysregulation of DNA methylation occurs in many cancer cells, in which the hypermethylation of promoter CpG islands may inactivate some tumor suppressor genes. ${ }^{3,4}$ MethylCpG binding domain proteins (MBD) are considered central players in DNA methylation-dependent gene silencing. ${ }^{1-3,9-13}$ These proteins can bind methylated $\mathrm{CpG}$ and further recruit corepressors, such as histone deacetylases (HDAC), to establish silent chromatin, thus providing a link between DNA methylation and transcriptional repression. ${ }^{2,12-14}$

The MBD protein family consists of five members, namely, MeCP2, MBD1, MBD2, MBD3, and MBD4. ${ }^{2,11,12,15-19}$ All MBD proteins, except MBD3, exhibit an affinity for methylated DNA., ${ }^{92,20}$ For example, MeCP2 and MBD2 are capable of binding to a single symmetrically methylated CpG pair. ${ }^{3,14,16,21}$
The complex of MBD protein and methylated DNA comprises an asymmetric MBD monomer and a symmetric DNA duplex. $^{12}$ The MBD protein folds into an $\alpha / \beta$-sandwich structure with characteristic loops. ${ }^{12,22}$ Recognition of the methyl-CpG pair depends on the hydrophobic interactions between the methyl groups of 5-methylcytosines and a hydrophobic patch formed by five residues, Val-20, Arg-22, Tyl-34, Arg-44, and Ser-45. ${ }^{9,12,23}$ Subtle variations in this hydrophobic network might abolish the binding of the protein to methylated DNA. For example, the loss of a single hydroxyl group Y34F renders MBD3 incapable of binding to methylated DNA. $^{3,12,20}$ Among all known MBD proteins, MBD2 has the highest affinity for methylated DNA and the greatest capacity to differentiate between methylated and unmethylated DNA. ${ }^{3}$ On the basis of the binding property of the MBD2 protein, a series of assays for detecting DNA methylation was established, e.g., methylated-CpG island recovery assay, ${ }^{1} \mathrm{MBD}$-isolated genome sequencing, ${ }^{4}$ and methylated DNA precipitation. ${ }^{5}$

Received: November 12, 2013

Accepted: January 4, 2014

Published: January 4, 2014 
MBD proteins link DNA methylation with transcriptional repression via the recruitment of corepressors, such as HDAC, leading to chromatin condensation. ${ }^{12,24}$ In general, all MBD proteins, except MBD4, are associated with HDAC subunits as part of large multisubunit complexes. ${ }^{3} \mathrm{MeCP} 2$ is associated with a transcriptional repressor complex containing $\operatorname{Sin} 3$ and histone deacetylases in mammalian cells. ${ }^{14,24,25}$ MBD2 is associated with MBD3 to form an $\mathrm{Mi}-2 / \mathrm{NuRD}$ complex that contains a chromatin-remodeling ATPase, a histone deacetylase, and other subunits. ${ }^{25,26}$

The DNA methylation-dependent transcriptional repression mechanism as described above would not work if MBD proteins could not selectively bind to methylated DNA. Thus, the specific recognition of $\mathrm{MBD}$ proteins against methylated DNA is fundamental to DNA methylation-induced gene silencing. However, nontarget interactions between basic MBD proteins and negatively charged DNA may occur. For example, MBD2b ( $\mathrm{p} I=9.32$, estimated by DNAman software) positively charged at physiological conditions ( $\mathrm{pH} 7.4$ ) should bind unmethylated DNA through electrostatic interactions. Hence, multiple MBD proteins may simultaneously bind on an unmethylated DNA region, which can cooperatively enhance the binding of the proteins to unmethylated DNA and attenuate the recognition specificity of the protein against methylated DNA.

In this study, we used fast capillary electrophoresis coupled with laser-induced fluorescence (CE-LIF) to investigate the interactions of MBD2b protein and double stranded (ds) DNA to pinpoint their binding stoichiometry and recognition specificity. CE-LIF is a powerful bioanalytical technology for the study of protein-DNA binding because of its high speed, high separation efficiency, high sensitivity, and low sample consumption. ${ }^{27}$ Moreover, CE-LIF has been exploited to study binding stoichiometry, dissociation kinetics, and conformational changes in biocomplexes. ${ }^{28-37}$ MBD2b protein was assayed on pairs of methylated and unmethylated doublestranded oligodeoxynucleotide probes with the adopted sequences from the $\mathrm{CpG}$ islands of $\mathrm{p} 16^{\mathrm{INK} 4 \mathrm{a}}$ gene.

\section{MATERIALS AND METHODS}

DNA Probes and MBD2b Protein. Single-stranded oligonucleotides were synthesized and purified by Shanghai Sangon Biological Engineering Technology and Services Co., Ltd. (Shanghai, China). These oligonucleotides are a part of the natural promoter $\mathrm{CpG}$ islands of the p16 ${ }^{\text {INK4a }}$ gene. The probes were labeled at their $5^{\prime}$ ends with tetramethylrhodamine (TMR) to facilitate the fluorescence detection of free DNA and DNA-protein complexes. All synthesized oligonucleotides have both methylated and unmethylated versions. To analyze systematically the effect of DNA length on the binding assay, dsDNA probes of different lengths were obtained by annealing two complementary strands, including 20mer*, 25mer*, 40mer*, 60mer*, and $80 \mathrm{mer}^{*}$. The detailed sequences are given in Supplementary Table $\mathrm{S} 1$ in the Supporting Information. The sequence of the 80mer* is as follows:

Forward: 5'TTCGGCTGACTGGCTGGCCA/CGGCCGC G G C C C G G G G T C G G / G T A G A G G A G G T G C GGGCGCT/GCTGGAGGCGGGGGCGCTGC 3';

reverse: 5' G C A G C G C C C C C G C C T C C A G C/ AGCGCCCGCACCTCCTCTAC/CCGACCCCGGGCCGCGGCCG/TGGCCAGCCAGTCAGCCGAA 3'.

Moreover, different methyl-CpG densities of 80 mer* were $^{*}$ designed: a nonmethylated version as control (U-80mer*), a monomethylated oligo with the methyl-CpG at the fifth $\mathrm{CpG}$ (M-80mer*), dimethylated oligos with the methyl-CpG at the second and seventh CpGs (D-80mer*), tetramethylated oligos with the methyl-CpGs at the position 2,6,7,9 (T-80mer*), and hexamethylated oligos with the methyl-CpGs at the position 2,5,6,7,9,10 (H-80mer*). The TMR-labeled methylated probes are summarized in Scheme 1. The single-stranded DNAs and

Scheme 1. Illustration of the Location of a Pair of Methylated CpG (O) in Synthetic Double-Stranded Oligodeoxynucleotide Probes ${ }^{a}$

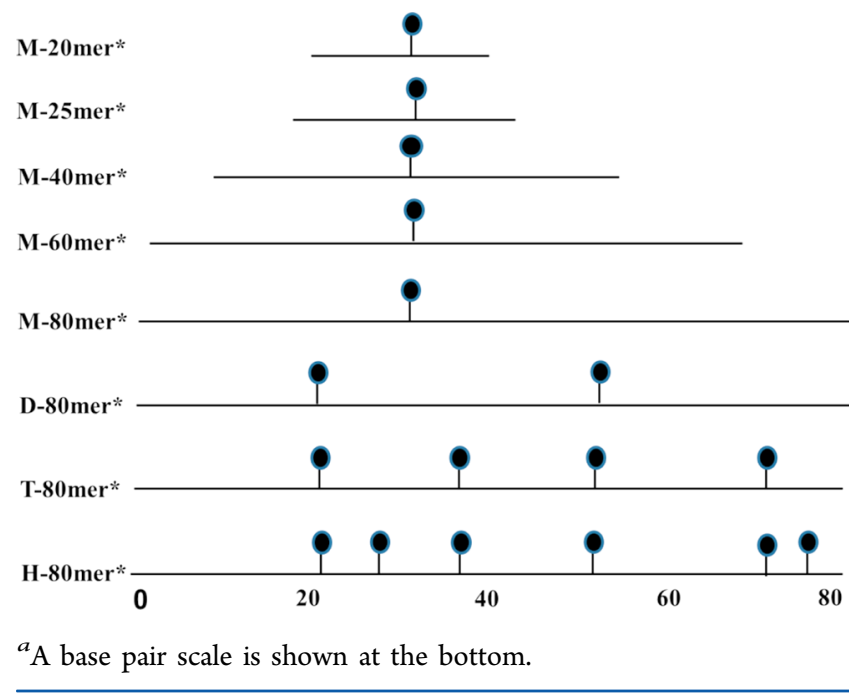

their complementary oligonucleotides were mixed at equimolar concentrations, annealed by bringing the solution to $95^{\circ} \mathrm{C}$, and allowed to cool down slowly to room temperature. The dsDNAs were purified and quantified as previously described. ${ }^{38}$ Prior to use, they were diluted into appropriate concentrations with a sample buffer of $1 \times$ TG, pH 8.5 (30 mM Tris and 160 $\mathrm{mM}$ glycine).

The recombinant MBD2b protein was constructed in our laboratory by gene engineering technology and was then purified by histidine-tagged affinity chromatography and cation ion exchange chromatography. The detailed protocol was described elsewhere. ${ }^{39}$

CE-LIF Analysis of Protein-DNA Interactions. CE analysis was performed on a home-built CE-LIF system as previously described. ${ }^{27,35,40}$ Fluorescence was excited by a helium-neon laser (543.5 nm, $1 \mathrm{~mW}$; Melles Griot, Irvine, CA) and detected by a photomultiplier tube (PMT, model R1477, Hamamatsu Photonics, Japan) at $575 \mathrm{~nm}$. The current signal output from the PMT was transformed to voltage signals with a current-to-voltage converter and handled by an HW2000 chromatographic workstation (Qianpu Software Co., Shanghai, China).

Prior to CE analysis, stock solutions of TMR-labeled DNA probes and protein were diluted to an appropriate concentration in a buffer of $1 \times \mathrm{TG} \mathrm{pH} 8.5$ and kept on ice. Different lengths and versions of probes (fixed at $2.0 \mathrm{nM}$ ) were mixed with varying protein concentrations $(0 \mathrm{nM}$ to $8.0 \mathrm{nM})$ in a reaction mixture containing $1 \times \mathrm{TG} \mathrm{pH} 8.5$, 5\% glycerol, 0.4 $\mathrm{mg} / \mathrm{mL}$ bovine serum albumin (BSA), $0.1 \%$ NP-40, $0.1 \mathrm{mM}$ EDTA, and $1 \mathrm{mM}$ DTT. The samples (typically $20 \mu \mathrm{L}$ ) were gently vortexed for $5 \mathrm{~s}$ and incubated on ice for $2 \mathrm{~h}$ before CELIF analysis. CE separation was conducted using a running buffer of $2 \times$ TGA (14 mM Tris, $108 \mathrm{mM}$ glycine, $10.5 \mathrm{mM}$ 
A)

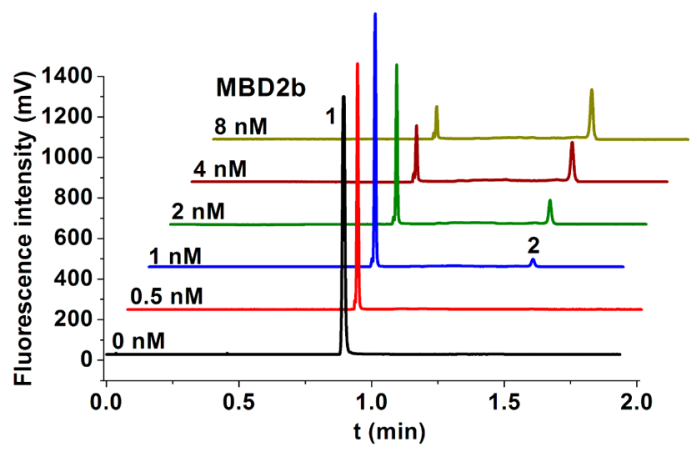

C)

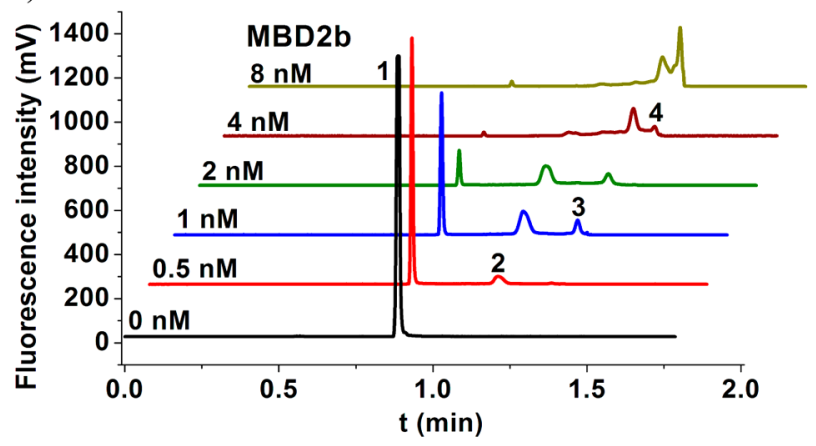

B)

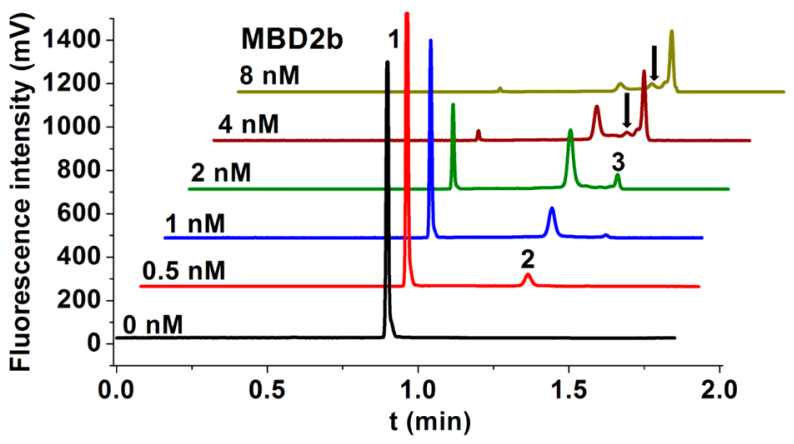

D)

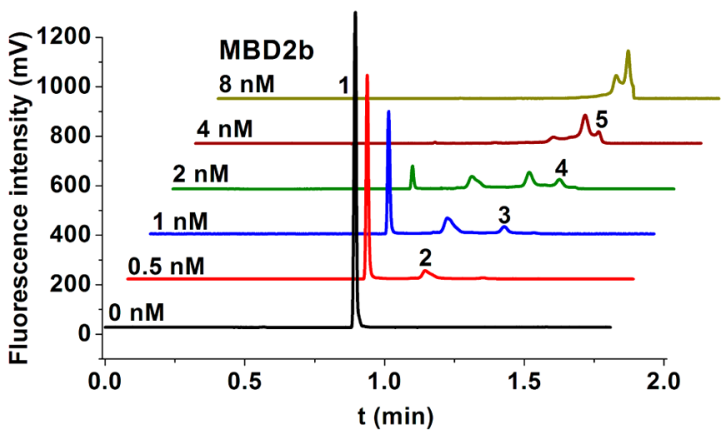

Figure 1. Capillary electrophoresis analysis of mixtures containing TMR-labeled DNA probes $(2.0 \mathrm{nM})$ and MBD2b protein $(0-8.0 \mathrm{nM})$, showing the presence of DNA-MBD2b complexes and free DNA probes. CE separation was performed with PAA coated capillary $(27.5 \mathrm{~cm}$ long, $50 \mu \mathrm{m}$ i.d., and $375 \mu \mathrm{m}$ o.d.) and $2 \times$ TGA (14 mM Tris, $108 \mathrm{mM}$ glycine, $\sim 10.5 \mathrm{mM} \mathrm{HAC}, \mathrm{pH} 7.5)$ buffer. Laser-induced fluorescence $\left(\lambda_{\mathrm{ex}}=543.5 \mathrm{~nm}\right.$ and $\lambda_{\mathrm{em}}$ $=575 \mathrm{~nm}$ ) was detected from the TMR-labeled species. Peak 1 is the free DNA, and peaks 2-5 correspond to the DNA-MBD2b complexes with corresponding stoichiometries of 1:1, 1:2, 1:3, and 1:4: (A) M-20mer*, (B) M-40mer*, (C) M-60mer*, and (D) M-80mer*. The black arrows (in part B) pointed at the peak that was probably formed by the dissociation of the DNA-MBD2b complex of 1:2 during CE separation. The observation of its migration between the peaks of DNA-MBD2b complexes of 1:1 and 1:2 supports the dissociation of the DNA-MBD2b complex of $1: 2$.

HAc, $\mathrm{pH}$ 7.5). The designed discontinuous buffer (TG-TGA) not only allows the focusing of the complexes but also enhances the formation and stability of previously described complexes. ${ }^{40}$ All the buffers were filtered through $0.22 \mu \mathrm{m}$ pore-size ultrafilters.

The inner surface of a fused-silica capillary with $50 \mu \mathrm{m}$ round i.d. and $365 \mu \mathrm{m}$ o.d. (Yongnian Optic Fiber Plant, Hebei, China) was coated with a monolayer of linear polyacrylamide (PAA) as previously described. ${ }^{41}$ The capillary was $27.5 \mathrm{~cm}$ long with an effective length of $21.5 \mathrm{~cm}$ from the inlet to the detection window. Samples were electrokinetically injected into the capillary by applying a voltage of $-20 \mathrm{kV}$ for $10 \mathrm{~s}$. Separations were conducted at room temperature. After each run, the capillary was washed by the running buffer for $4 \mathrm{~min}$.

Calculation of Binding Parameters. Specifically, we adopted a nonequilibrium affinity capillary electrophoresis. ${ }^{28-33}$ If the binding of protein (P) to DNA can be simplified as a second-order reaction $\mathrm{P}+\mathrm{DNA} \rightarrow \mathrm{DNA} \cdot \mathrm{P}$, then the apparent dissociation constants $\left(K_{\mathrm{d}}\right)$ and apparent dissociation rate constants $\left(k_{\text {off }}\right)$ of the complexes can be calculated by the following equations as described for the nonequilibrium affinity capillary electrophoresis: ${ }^{30-33}$

$$
\begin{aligned}
& K_{\mathrm{d}}=\frac{[\mathrm{P}]_{0}(1+C /(A+B))-[\mathrm{DNA}]_{0}}{1+(A+B) / C} \\
& k_{\text {off }}=\ln \left(\frac{A+B}{A}\right) / t_{\text {DNA.P }}
\end{aligned}
$$

$A, B$, and $C$ correspond to the peak areas of the undissociated DNA-protein complex, the DNA probe dissociated from the complex during CE separation, and the unbound DNA probe, respectively. $[\mathrm{P}]_{0}$ and $[\mathrm{DNA}]_{0}$ are the total concentrations of protein and DNA in the sample, respectively, and $t_{\text {DNA.P }}$ is the migration time of the DNA-protein complex.

\section{RESULTS AND DISCUSSION}

Length-Dependent Stoichiometry of DNA-MBD2b Complexes. To characterize the stoichiometry of the MBD2b protein binding to methylated dsDNA, a set of double-stranded oligonucleotide probes of different length was synthesized. These probes contain a single pair of methylatedCpG sites and a TMR label at the $5^{\prime}$ end, including M-20mer*, M-40mer*, M-60mer*, and M-80mer* probes (Scheme 1; M indicates one pair of methylated $\mathrm{CpG}$, and $*$ indicates the TMR label). The single methylated $\mathrm{CpG}$ pair is located at the central position of the DNA probes to ensure that the protein establishes all the contacts required for its correct positioning along the duplex. The methylated $\mathrm{CpG}$ pair in the probes functioned as a specifically recognizable site for MBD2b protein, whereas the TMR served as a suitable label for measuring fluorescence intensity. Correspondingly, the unmethylated versions of these probes were used to evaluate the contributions of the nonmethylated part of DNA to the tested bindings.

Figure 1 shows a series of electropherograms from the CELIF analyses of mixtures containing one of the TMR-labeled 
methylated-DNA probes of $2.0 \mathrm{nM}$ (M-20mer*, M-40mer*, M$60 \mathrm{mer}^{*}$, and M-80mer*) and MBD2b protein of varying concentrations ( $0 \mathrm{nM}$ to $8.0 \mathrm{nM})$. Only peak 1 was observed when no MBD2b protein was present in the binding solution, indicating that peak 1 represents a free DNA probe. The migration times of the free DNA probes of different lengths are almost similar ( $\sim 0.86 \mathrm{~min})$, suggesting identical charge-to-mass ratios. $^{29}$ The addition of the MBD2b protein to the TMRlabeled dsDNA probe-contained solutions produced slower migrating peaks (peaks 2-5) depicting complexes of different stoichiometries, accompanied with the decrease in the free DNA probes (peak 1). Under our conditions, all the complexes (after $1.05 \mathrm{~min}$ ) were well separated from the free DNA probes $(\sim 0.86 \mathrm{~min})$, and all the separations were completed within $1.45 \mathrm{~min}$, suggesting the fast and effective separation of proteinbound and unbound DNA by CE.

As shown in Figure 1A, the MBD2b protein forms only one complex with the $\mathrm{M}-20 \mathrm{mer} *$ in all the tested protein concentrations $(0.5-8.0 \mathrm{nM})$. The complexes formed at different concentrations of MBD2b protein exhibit similar migration times $(\sim 1.45 \mathrm{~min})$. Considering that the MBD2b protein is the only monomer that binds methylated DNA, ${ }^{12}$ we believe that this observed DNA-MBD2b complex has a stoichiometry of $1: 1$. Thus, one MBD2b protein binds one $\mathrm{M}$ 20mer*. The binding equation is depicted as follows:

$$
\mathrm{P}+20 \mathrm{mer}^{*}=\mathrm{P}\left(20 \mathrm{mer}^{*}\right)
$$

where $\mathrm{P}$ represents a monomer of the MBD2b protein.

Interestingly, two complexes were observed when the length of the DNA probe was increased to $40 \mathrm{bp}$ (M-40mer*, Figure 1B). The formation of the two complexes is dependent on the concentration of the $\mathrm{MBD} 2 \mathrm{~b}$ protein. When the protein concentration was below $1.0 \mathrm{nM}$, one complex (peak 2, $\sim 1.28$ min) first appeared with a decrease in free DNA probe (peak 1, $\sim 0.86 \mathrm{~min})$. Upon further increase in the MBD2b concentration $(2.0-8.0 \mathrm{nM}$ ), another complex (peak 3, 1.43 min) appeared. The second complex became predominant at a high concentration of MBD2b (8.0 nM). Meanwhile, free M-40mer* gradually disappeared (Figure 1B). The two peaks likely corresponded to the complexes of M-40mer*-MBD2b with a stoichiometry of 1:1 (peak 2, 1.28 min) and 1:2 (peak 3, $\sim 1.43 \mathrm{~min}$ ). The small peaks between the $1: 1$ and $1: 2$ complexes (indicated by black arrows) observed at a protein concentration of 4.0 and $8.0 \mathrm{nM}$ are probably caused by the dissociation of the complex of 1:2. In this case, the binding equations are expected as follows:

$$
\begin{aligned}
& \mathrm{P}+40 \text { mer }^{*}=\mathrm{P}\left(40 \text { mer }^{*}\right) \\
& \mathrm{P}+\mathrm{P}\left(40 \text { mer }^{*}\right)=\mathrm{P}_{2}\left(40 \text { mer }^{*}\right)
\end{aligned}
$$

Similarly, three complexes of distinct stoichiometries formed upon the incubation of the MBD2b protein with an M-60mer* probe (Figure 1C). Peaks 2 to 4 are correspondingly attributed to the 1:1 complex (peak 2, 1.13 min), 1:2 complex (peak 3, $\sim 1.31 \mathrm{~min}$ ), and 1:3 complex (peak $4, \sim 1.40 \mathrm{~min}$ ). The shift of the binding stoichiometry from $1: 1$ to $1: 3$ is MBD2b concentration-dependent. The binding equations are shown as follows:

$$
\begin{aligned}
& \mathrm{P}+60 \text { mer }^{*}=\mathrm{P}\left(60 \text { mer }^{*}\right) \\
& \mathrm{P}+\mathrm{P}\left(60 \text { mer }^{*}\right)=\mathrm{P}_{2}\left(60 \text { mer }^{*}\right) \\
& \mathrm{P}+\mathrm{P}_{2}\left(60 \text { mer }^{*}\right)=\mathrm{P}_{3}\left(60 \text { mer }^{*}\right)
\end{aligned}
$$

Accordingly, an M-80mer* probe exhibits the maximal capacity of accommodating four MBD2b molecules. Indeed, all four complexes with respective stoichiometries of 1:1 (peak 2), 1:2 (peak 3), 1:3 (peak 4), and 1:4 (peak 5) were observed by changing the MBD2b concentration (Figure 1D). When the MBD2b protein concentration was $2.0 \mathrm{nM}$, all four complexes appeared simultaneously. The complexes of $1: 1,1: 2,1: 3$, and $1: 4$ sequentially migrated out of the capillary with a migration time of $1.07,1.28,1.38$, and $1.44 \mathrm{~min}$, respectively. The binding equations are depicted as follows:

$$
\begin{aligned}
& \mathrm{P}+80 \text { mer }^{*}=\mathrm{P}\left(80 \text { mer }^{*}\right) \\
& \mathrm{P}+\mathrm{P}\left(80 \text { mer }^{*}\right)=\mathrm{P}_{2}\left(80 \text { mer }^{*}\right) \\
& \mathrm{P}+\mathrm{P}_{2}\left(80 \text { mer }^{*}\right)=\mathrm{P}_{3}\left(80 \text { mer }^{*}\right) \\
& \mathrm{P}+\mathrm{P}_{3}\left(80 \text { mer }^{*}\right)=\mathrm{P}_{4}\left(80 \text { mer }^{*}\right)
\end{aligned}
$$

Thus, a self-consistent pattern of binding stoichiometry was established. The M-20mer* has one binding site, M-40mer* has two sites, M-60mer* has three sites, and M-80mer* is long enough to accommodate four MBD2b molecules (Scheme 2). Each MBD2b protein occupies $\sim 20$ bp nucleotides in DNA.

Scheme 2. Length-Dependent Binding Stoichiometry of the MBD2b Protein with Methylated DNA

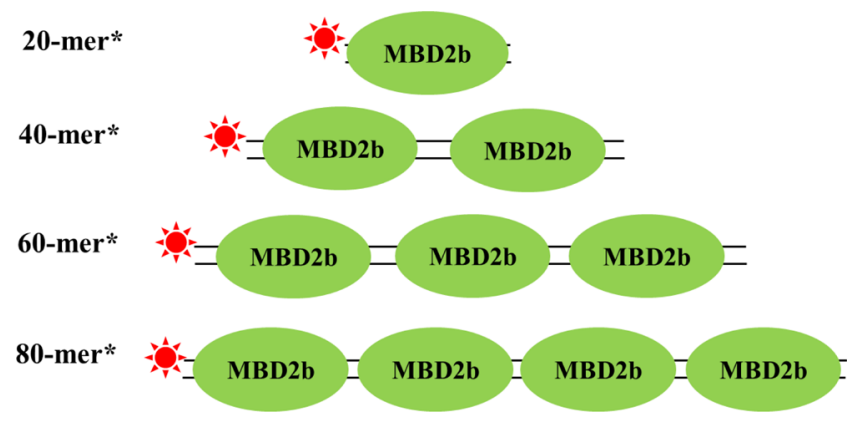

Given that one MBD2b protein occupies $\sim 20$ bp nucleotides of DNA, DNA with a length between $20 \mathrm{bp}$ and $40 \mathrm{bp}$ is expected to have one MBD2b binding site but is insufficient to afford the second binding site. Indeed, only the 1:1 complex formed for $25 \mathrm{bp}$ dsDNA, as shown in Supplementary Figure 1 in the Supporting Information (peak 2, 1.43 min), further confirming our conclusion on the binding stoichiometry of DNA and MBD2b.

Methylation Density-Independent Binding Stoichiometry. Interestingly, all of the above tested probes that contain only a single methyl-CpG pair each exhibit lengthdependent stoichiometry to the MBD2b protein binding. For example, one M-80mer* molecule maximally binds four MBD2b proteins, indicating that the binding stoichiometry is not determined by the methylation of the CpG pair in the tested DNA probes.

We then tested the binding of the MBD2b protein to DNA containing different numbers of methylated $\mathrm{CpG}$ pairs through a set of 80 bp DNA probes, including U-80mer* (ummethylated), M-80mer* (one pair of methylated CpG), D-80mer* (two pairs of methylated $\mathrm{CpG}$ ), T-80mer* (four pairs of methylated $\mathrm{CpG}$ ), and $\mathrm{H}-80 \mathrm{mer}^{*}$ (six pairs of methylated CpG).

All 80 bp DNA probes (U-80mer*, M-80mer*, D-80mer*, T-80mer*, and H-80mer*) display similar binding stoichiom- 
etry patterns (from 1:2 to $1: 4$ ) regardless of their number of methylated CpG pairs (Figure 2). Unmethylated 80 bp DNA
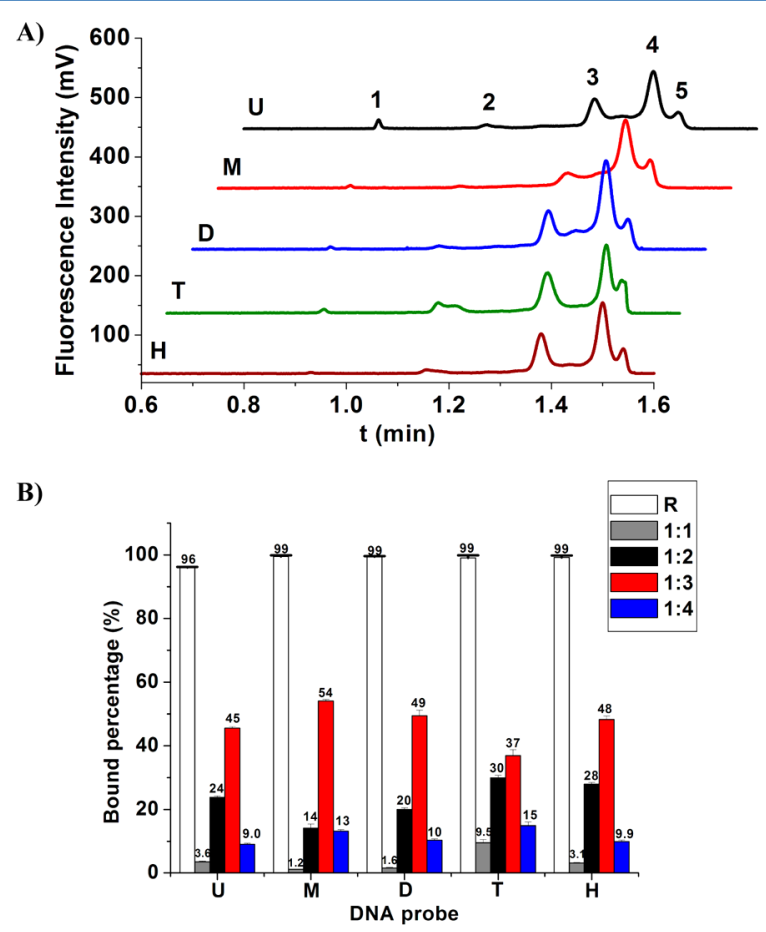

Figure 2. (A) CE-LIF analysis of mixtures containing 80mer* probes $(2.0 \mathrm{nM})$ of different methyl-CpG densities and MBD2b protein $(4.0$ nM) U, U-80mer*; M, M-80mer*; D, D-80mer*; T, T-80mer*; H, H80mer*.The same conditions as shown in Figure 1 were used for analyses. (B) The estimated binding percentage for each DNAMBD2b complexes and total binding percentage $(R)$.

probes (U-80mer*) also display a similar stoichiometry pattern (Figure 2A). Consistently, the complex of $80 \mathrm{mer}^{*}-\mathrm{MBD} 2 \mathrm{~b}$ of $1: 3$ (binding percentage, $37 \%-54 \%$ ) dominates among three observed complexes (Figure 2A,B). The maximal binding number of MBD2b on $80 \mathrm{bp}$ DNA is independent of DNA methylation.

The binding percentages of all $80 \mathrm{bp}$ DNA probes are similar (96-99\%) regardless of their number of methylated CpG pairs (Figure 2B). Unmethylated $80 \mathrm{bp}$ DNA probes (U-80mer*) also display a high binding percentage ( $96 \%$, Figure $2 B)$. In this case, the MBD2b protein cannot discriminate the methylated from unmethylated DNA.

Simultaneous Binding of Multiple MBD2b Proteins to DNA Attenuates Recognition Specificity against Methylated DNA. To understand the recognition specificity of the $\mathrm{MBD} 2 \mathrm{~b}$ protein against methylated DNA, we further compared monomethylated DNA probes with the unmethylated DNA probes of different lengths ( $20 \mathrm{bp}$ to $80 \mathrm{bp}$, Scheme 1) in terms of MBD2b protein binding specificity.

The short probes ( $20 \mathrm{bp}$ to $25 \mathrm{bp}$ ) can bind only one $\mathrm{MBD} 2 \mathrm{~b}$ protein (Figures 1 and 3 ). The $\mathrm{MBD} 2 \mathrm{~b}$ protein prefers binding to the monomethylated short probes (Figure 3A,B). The monomethylated probes are bound to MBD2b by $35.4 \%$ to $64.0 \%$ (Figure 3C). By contrast, the unmethylated probes are bound to MBD2b only by $11.3 \%$ to $25.6 \%$ (Figure 3C). The estimated ratios of the binding percentage of the short unmethylated probes $\left(R_{\mathrm{U}}\right)$ to those of the monomethylated probes $\left(R_{\mathrm{M}}\right)$ range from $\sim 0.32$ to 0.35 (Figure $3 \mathrm{D}$ ). The estimated affinity of MBD2b with unmethylated 20mer* and
25 mer* $^{*}$ is 5.3 - to 10.6 -fold lower than that with methylated 20mer* and 25mer* (Figure 3E). The MBD2b protein can bind methylated short oligonucleotides with high affinity $\left(K_{\mathrm{d}}\right.$ : $0.39-2.29 \mathrm{nM}$ ). These results consistently suggest that the MBD2b protein can specifically recognize methylated CpG (with high affinity) in short oligodeoxynucleotides ( $\leq 25 \mathrm{bp}$ ).

However, long DNA probes (40-80 bp) can bind more than one MBD2b protein (Figures 1 and 3 ). Both the monomethylated and unmethylated probes are effectively bound to MBD2b (Figure 3A,B). The monomethylated probes are bound to MBD2b by $74.8 \%$ to $88.6 \%$, slightly higher than that for the unmethylated probes (58.5-76.6\%) (Figure 3C). The estimated ratios of $R_{\mathrm{U}}$ to $R_{\mathrm{M}}\left(R_{\mathrm{U}} / R_{\mathrm{M}}\right)$ are about $0.76-0.86$ (Figure 3D). By contrast, the $R_{\mathrm{U}} / R_{\mathrm{M}}$ values for short DNA probes (20mer* and 25mer*) are much lower (0.32-0.35). The abrupt increase in $R_{\mathrm{U}} / R_{\mathrm{M}}$ for long DNA probes ( $\geq 40 \mathrm{bp}$ ) suggests the reduced methylation-specific binding of the MBD2b protein to DNA.

Hence, only one MBD2b protein molecule can bind a shorter DNA molecule (20 bp to $25 \mathrm{bp}$ ) with excellent recognition specificity to discriminate methylated and unmethylated DNA. However, multiple MBD2b proteins can bind a longer DNA molecule ( $40 \mathrm{bp}$ to $80 \mathrm{bp}$ ) with poor discrimination ability for methylated and unmethylated DNA. Therefore, the DNA length-dependent binding stoichiometry and multiple MBD2b protein binding probably attenuate the recognition specificity of $\mathrm{MBD} 2 \mathrm{~b}$ protein against methylated DNA. Hence, the multiple binding of MBD2b protein to DNA cooperatively enhances nonspecific interactions with the unmethylated DNA. The nonspecific binding is probably due to the electrostatic interactions between the positively charged residues of $\mathrm{MBD} 2 \mathrm{~b}(\mathrm{pI}=9.32)$ and the DNA phosphate backbone. ${ }^{42-44}$

DNA Length-Dependent Dissociation Kinetics of MBD2b Protein-DNA Interaction. Broad plateaus (labeled by red color and indicated by red arrow) are observed between the two peaks of the DNA-MBD2b complex of 1:1 (peak 2) and unbound TMR-labeled probe (peak 1, 20-mer*, 25-mer*), as shown by Figure $4 A, B$, which are adapted from amplified Figure 3A,B. These broad zones (Figure 4A,B) are completely different from the narrow, approximately symmetric Gaussian peaks 2, which are considered the undissociated and stable DNA-MBD2b complexes of $1: 1$. We attribute those broad zones to the unique zone formed by the dissociation of the DNA-MBD2b complex of 1:1 during CE separation. Evidently, the dissociation zones are predominant for the unmethylated probes (20mer* and $25 \mathrm{mer}^{*}$ ). By contrast, the stable and undissociated DNA-MBD2b complexes of 1:1 (peaks 2) are predominant for all the monomethylated probes (20mer*, 25mer*, and 40mer*).

Considering the observed dissociation zone as previously described, ${ }^{30-33}$ we quantitatively estimated the dissociation rate constants of the DNA-MBD2b complexes of 1:1 (Figure 4C). The measured dissociation rate constants for the unmethylated and monomethylated probes $\left(20 \mathrm{mer}^{*}, 25 \mathrm{mer}^{*}\right.$, and $\left.40 \mathrm{mer}^{*}\right)$ are approximately $(0.42$ to 2.74$) \times 10^{-2}$ and $(0.02$ to 1.00$) \times$ $10^{-2} \mathrm{~s}^{-1}$, respectively. The dissociation rate constants for the monomethylated probes are 1.67 - to 20 -fold lower than those for the unmethylated probes (Figure 4C). The increase in the length of DNA probes (40mer*) reduced the dissociation rate constants $\left(k_{\text {off }}\right)$ for both the unmethylated and methylated probes. However, the methylated DNA probes displayed more 



Figure 3. CE-LIF analysis of mixtures containing unmethylated and monomethylated DNA probes (2.0 nM) and MBD2b protein (2.0 nM), showing attenuated specificity by multiple MBD2b binding: (A) unmethylated probes, (B) methylated probes, (C) binding percentage $R$ of each DNA probe with MBD2b. (D) $R_{\mathrm{U}} / R_{\mathrm{M}}$ calculated by data obtained from part C. (E) Dissociation constant $\left(K_{\mathrm{d}}\right.$, $\left.\mathrm{nM}\right)$ for $20 \mathrm{mer} *$ and $25 \mathrm{mer}$ *

significant reduction in $k_{\text {off }}$, e.g. the $k_{\text {off }}$ for methylated $40 \mathrm{mer} *$ is about 20 -fold lower than that for unmethylated $40 \mathrm{mer}^{*}$.

These data suggest that the complexes of unmethylated DNA-MBD2b (1:1) easily dissociate. Hence, the DNAMBD2b complexes of 1:1 for methylated DNA are more stable than that for unmethylated DNA.

\section{CONCLUSIONS}

In summary, we demonstrated a detailed study of the interplay of stoichiometry and recognition specificity for the interaction of one MBD protein and methylated DNA. The binding stoichiometry of the $\mathrm{MBD} 2 \mathrm{~b}$ protein depends on the length of
DNA, and multiple binding of MBD2b protein to one DNA molecule decreases the recognition specificity of $\mathrm{MBD} 2 \mathrm{~b}$ protein. Therefore, the binding stoichiometry of multiple MBD2b binding one DNA may compromise the DNA methylation-triggered recognition specificity. The study not only provides new insights into binding stoichiometry but also enhances the understanding of the methyl-specific recognition mechanism. These insights also facilitate the improvement of MBD protein-based assays for detecting DNA methylation. 

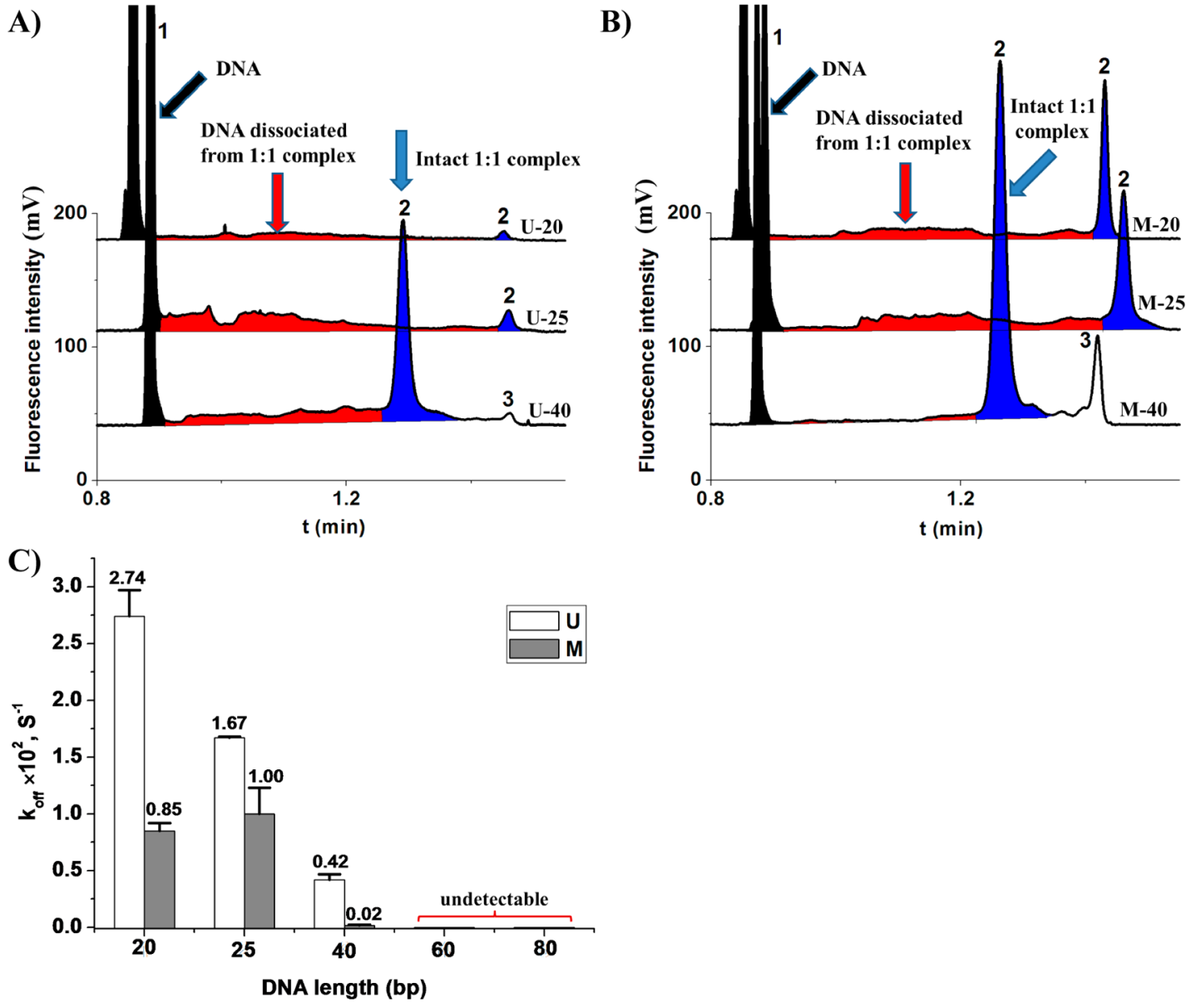

Figure 4. CE-LIF analysis of dissociation kinetics of DNA probes $(2.0 \mathrm{nM})$ complexed with MBD2b protein $(2.0 \mathrm{nM})$ : (A) U-20mer*, U-25mer*, U-40mer* probes and (B) M-20mer*, M-25mer* probes. (C) Measured apparent dissociation rate constants $\left(k_{\text {off }} \mathrm{s}^{-1}\right)$ for $1: 1$ complex.

\section{ASSOCIATED CONTENT}

\section{S Supporting Information}

Additional information as noted in text. This material is available free of charge via the Internet at http://pubs.acs.org.

\section{AUTHOR INFORMATION}

\section{Corresponding Author}

*E-mail: hlwang@rcees.ac.cn. Phone/fax: +86-10-62849600.

\section{Notes}

The authors declare no competing financial interest.

\section{ACKNOWLEDGMENTS}

This work was supported by the grants from the National Natural Science Foundation of China (Grants 21077129, 21125523, and 21300076) and the National Basic Research Program of China (Grants 2009CB421065 and 2011CB936001).

\section{REFERENCES}

(1) Rauch, T.; Pfeifer, G. P. Lab. Invest. 2005, 85, 1172-1180.

(2) Ballestar, E.; Paz, M. F.; Valle, L.; Wei, S.; Fraga, M. F.; Espada, J.; Cigudosa, J. C.; Huang, T. H. M.; Esteller, M. EMBO J. 2003, 22, $6335-6345$.

(3) Fraga, M. F.; Ballestar, E.; Montoya, G.; Taysavang, P.; Wade, P. A.; Esteller, M. Nucleic Acids Res. 2003, 31, 1765-1774.

(4) Serre, D.; Lee, B. H.; Ting, A. H. Nucleic Acids Res. 2010, 38, 391-399.
(5) Oliver, V. F.; Wan, J.; Agarwal, S.; Zack, D. J.; Qian, J.; Merbs, S. L. Epigenet. Chromatin. 2013, 6, 17-27.

(6) Portela, A.; Esteller, M. Nat. Biotechnol. 2010, 28, 1057-1068.

(7) Yin, H.; Zhou, Y.; Xu, Z.; Chen, L.; Zhang, D.; Ai, S. Biosens. Bioelectron. 2013, 41, 492-497.

(8) Yin, R.; Mao, S.-Q.; Zhao, B.; Chong, Z.; Yang, Y.; Zhao, C.; Zhang, D.; Huang, H.; Gao, J.; Li, Z.; Jiao, Y.; Li, C.; Liu, S.; Wu, D.; Gu, W.; Yang, Y.-G.; Xu, G.-L.; Wang, H. J. Am. Chem. Soc. 2013, 135, 10396-10403.

(9) Födermayr, M.; Pröll, J.; Zach, O.; Wechselberger, C.; Lutz, D. Mol. Biol. Rep. 2009, 36, 1859-1862.

(10) Klose, R. J.; Bird, A. P. Trends Biochem. Sci. 2006, 31, 89-96.

(11) Clouaire, T.; Heras, J. I.; Merusi, C.; Stancheva, I. Nucleic Acids Res. 2010, 38, 4620-4634.

(12) Clouaire, T.; Stancheva, I. Cell. Mol. Life Sci. 2008, 65, 15091522.

(13) Ballestar, E.; Esteller, M. Carcinogenesis 2002, 23, 1103-1109.

(14) Wade, P. A. Oncogene 2001, 20, 3166-3173.

(15) Lopez-Serra, L.; Ballestar, E.; Fraga, M. F.; Alaminos, M.; Setien, F.; Esteller, M. Cancer Res. 2006, 66, 8342-8346.

(16) Ballestar, E.; Wolffe, A. P. Eur. J. Biochem. 2001, 268, 1-6.

(17) Nan, X.; Meehan, R. R.; Bird, A. Nucleic Acids Res. 1993, 21, 4886-4892.

(18) Dhasarathy, A.; Wade, P. A. Mutat. Res. 2008, 647, 39-43.

(19) Prokhortchouk, E.; Defossez, P.-A. Biochim. Biophys. Acta 2008, 1783, 2167-2173.

(20) Sasai, N.; Defossez, P.-A. Int. J. Dev. Biol. 2009, 53, 323-334.

(21) Fuks, F.; Hurd, P. J.; Wolf, D.; Nan, X.; Bird, A. P.; Kouzarides, T. J. Biol. Chem. 2003, 278, 4035-4040.

(22) Ohki, I.; Shimotake, N.; Fujita, N.; Nakao, M.; Shirakawa, M. EMBO J. 1999, 18, 6653-6661. 
(23) Ohki, I.; Shimotake, N.; Fujita, N.; Jee, J.-G.; Ikegami, T.; Nakao, M.; Shirakawa, M. Cell 2001, 105, 487-497.

(24) Hansen, J. C.; Ghosh, R. P.; Woodcock, C. L. IUBMB Life 2010, $62,732-738$.

(25) Jiang, C.-L.; Jin, S.-G.; Pfeifer, G. P. J. Biol. Chem. 2004, 279, 52456-52464.

(26) Guezennec, X. L.; Vermeulen, M.; Brinkman, A. B.; Hoeijmakers, W. A. M.; Cohen, A.; Lasonder, E.; Stunnenberg, H. G. Mol. Cell. Biol. 2006, 26, 843-851.

(27) Wang, H.; Xing, J.; Tan, W.; Lam, M.; Carnelley, T.; Weinfeld, M.; Le, X. C. Anal. Chem. 2002, 74, 3714-3719.

(28) Petrov, A.; Okhonin, V.; Berezovski, M.; Krylov, S. N. J. Am. Chem. Soc. 2005, 127, 17104-17110.

(29) Berezovski, M.; Drabovich, A.; Krylova, S. M.; Musheev, M.; Okhonin, V.; Petrov, A.; Krylov, S. N. J. Am. Chem. Soc. 2005, 127, 3165-3171.

(30) Krylov, S. N. Electrophoresis 2007, 28, 69-88.

(31) Berezovski, M.; Krylov, S. N. J. Am. Chem. Soc. 2002, 124, 13674-13675.

(32) Krylov, S. N.; Berezovski, M. Analyst 2003, 128, 571-575.

(33) Berezovski, M.; Krylov, S. N. Anal. Chem. 2005, 77, 1526-1529.

(34) Wang, H.; Lu, M.; Tang, M.; Houten, B. V.; Ross, J. B. A.; Weinfeldf, M.; Le, X. C. Proc. Natl. Acad. Sci. U.S.A. 2009, 106, 1284912854.

(35) Wang, H.; Lu, M.; Mei, N.; Lee, J.; Weinfeld, M.; Le, X. C. Anal. Chim. Acta 2003, 500, 13-20.

(36) Li, T.; Wang, H. Anal. Chem. 2009, 81, 1988-1995.

(37) Berezovski, M.; Nutiu, R.; Li, Y.; Krylov, S. N. Anal. Chem. 2003, $75,1382-1386$.

(38) Wang, C.; Feng, F.; Wang, Z.; Li, T.; Le, X. C.; Wang, H. Chem. Res. Toxicol. 2009, 22, 676-682.

(39) Zou D.; Wang X.; Chen Z.; Zhang, D.; Wang, H. Sci. China Chem. 2014, Manuscript ID SCC-2013-0288.

(40) Wang, H.; Lu, M.; Le, X. C. Anal. Chem. 2005, 77, 4985-4990. (41) Wang, Z.; Wang, C.; Yin, J.; Song, M.; Lu, M.; Wang, H. Electrophoresis 2008, 29, 4454-4462.

(42) Kalodimos, C. G.; Biris, N.; Bonvin, A. M. J. J.; Levandoski, M. M.; Guennuegues, M.; Boelens, R.; Kaptein, R. Science 2004, 305, 386-389.

(43) Cherstvy, A. G.; Kolomeisky, A. B.; Kornyshev, A. A. J. Phys. Chem. B 2008, 112, 4741-4750.

(44) Ganguly, A.; Rajdev, P.; Williams, S. M.; Chatterji, D. J. Phys. Chem. B 2012, 116, 621-632. 